\title{
ene
}

\section{FACTORES RELACIONADOS CON EL LUGAR DE FALLECIMIENTO EN CUIDADOS PALIATIVOS PEDIÁTRICOS}

FACTORS RELATED TO THE PLACE OF DEATH OF PATIENTS IN A PEDIATRIC PALLIATIVE CARE UNIT. WHAT DOES THE PLACE OF DEATH OF THE CHILDREN DEPEND ON?

Ciprés Roig, S; Gutiérrez Rada, C.

Unidad de Cuidados Paliativos Pediátricos. Hospital Sant Joan de Déu, Esplugues de Llobregat, Barcelona. 


\section{Resumen}

Introducción:

Los cuidados paliativos pediátricos pretenden responder a las necesidades que presentan niños con enfermedades amenazantes o limitantes para la vida y sus familias siempre desde un abordaje bio-psico-social y espiritual.

En la unidad de cuidados paliativos pediátricos del hospital Sant Joan de Déu se atiende a estos pacientes allí donde se encuentren. La tendencia y prevalencia del lugar donde fallecen los niños atendidos por la unidad es variable con el tiempo y depende de diversos factores.

Objetivo:

Describir qué factores se asocian al lugar de fallecimiento en una población de pacientes atendidos por la unidad de cuidados paliativos pediátricos.

Metodología:

Estudio descriptivo transversal y retrospectivo con población atendida por la unidad de cuidados paliativos pediátricos del hospital Sant Joan de Déu entre los años 2010 y 2015. La muestra incluyó al total de niños y familias atendidos en dicho periodo, y quedaron excluidos aquellos pacientes que no fallecieron durante el mismo. Se revisaron variables como la especialidad de derivación, el tiempo de seguimiento, el lugar donde se recibía la atención y el tipo de consulta provista, así como el emplazamiento del fallecimiento.

Resultados:

De los 198 niños incluidos, con edades comprendidas entre pocas horas de vida y 22 años, el 41,9\% fallecieron en su domicilio. Las especialidades de origen mayoritarias de los pacientes fueron oncología y neurología. La población estudiada estaba formada por niños con enfermedades graves, generalmente incurables y que limitan o amenazan su vida.

Existe correlación significativa positiva entre el tiempo de seguimiento y el número de consultas domiciliarias y telefónicas. Este periodo de seguimiento era significativamente mayor en aquellos que fallecieron en su domicilio.

En el análisis se encuentran diferencias estadísticamente significativas entre pacientes con patologías onco-hematológicas y no onco-hematológicas.

Conclusiones:

El tiempo de seguimiento de los pacientes y sus familias por la unidad de cuidados paliativos pediátricos está relacionado con el fallecimiento en domicilio.

\section{Palabras clave}

Cuidados paliativos, pediatría, cuidados al final de la vida, atención en domicilio. 


\section{Abstract}

\section{Background}

Pediatric palliative care aims to respond to the needs of children with lifethreatening and/or life-limiting diseases and their families from a bio-psycho-social and spiritual perspective. The pediatric palliative care unit of Sant Joan de Deu Hospitalis was designed to address these needs, both in hospital and at home. The trend and prevalence of where children die varies over time and depends on diverse features.

Objective

Describe the factors associated with the place of death in a population of patients seen by a Pediatric Palliative Care Unit.

\section{Methodology:}

Cross-sectional, retrospective observational, descriptive and correlational study of the patients attended by the unit of the Sant Joan de Deu Hospital between the years 2010 and 2015. The population includes all of the children treated by the unit in the above-mentioned period excluding those who exceeded their life expectancy.

Results

Out of the 198 studied patients aged from 0 days to 22 years - $41,9 \%$ died at home. The average of follow- up time was 184 days. Most of the cases were addressed from Oncology (50\%) and Neurology (28, 3\%).The present population consisted of children with severe illnesses, generally incurables and lifelimiting or life threatening.

It exists a high correlation between the follow-up time and the amount of telephone inquiry and home consultation. The remaining time at the unit was higher on children who died at home comparing to the hospital setting.

The analysis of the differences between onco-hematological or non onco-hematological illnesses led to the detection of a statistical significance between the different kind of consultation (at hospital, home or telematic ones).

\section{Conclusions}

The length of stay of patients in the pediatric palliative care unit is associated with the likelihood of death occurring at home.

\section{Keywords}

Palliative care, pediatrics, end-oflife care, place of death, home care. 


\section{INTRODUCCIÓN}

En las últimas décadas, los avances científico-técnicos han contribuido a disminuir la mortalidad infantil progresivamente cada año. Sin embargo, esta mejora conlleva un incremento de la prevalencia de pacientes pediátricos con enfermedades crónicas complejas. Éstos constituyen un colectivo frágil y vulnerable, igual que ocurre en el ámbito adulto, tributario de recibir cuidados paliativos (1).

Durante muchos años los cuidados paliativos no han estado al alcance de la edad pediátrica e incluso actualmente, cuando en Europa se están desarrollando programas de cuidados paliativos pediátricos (CPP) en diferentes países, como Noruega o Irlanda (20), tan sólo una minoría están diseñados para atender de forma apropiada las necesidades de estos niños. Es decir, existe una población pediátrica con una serie de necesidades que hoy no se encuentran adecuadamente cubiertas $(2,3)$.

La definición de CPP de la Organización Mundial de la Salud habla de unos cuidados activos totales del cuerpo, la mente y el espíritu del niño y la familia, que comienzan con el diagnóstico de una enfermedad amenazante o limitante para la vida, y continúan independientemente de si recibe o no tratamiento para la propia enfermedad (4). Para lograr ofrecer este tipo de cuidados de una forma óptima, los profesionales sanitarios deben evaluar y aliviar el sufrimiento físico, psicológico, espiritual y social del niño y su familia (5).

Unos cuidados paliativos efectivos requieren un amplio enfoque multidisciplinar que haga uso de los recursos disponibles en la comunidad; pueden llevarse a cabo con éxito incluso si los recursos son limitados y pueden prestarse tanto en centros terciarios, como en centros de salud comunitarios e incluso en el domicilio de los niños (6).

El hospital Sant Joan de Déu de Barcelona, es un hospital materno infantil de tercer nivel que atiende a pacientes con pluripatología de alta complejidad y dispone de una unidad de cuidados paliativos pediátricos (UCPP) desde el año 1991, en constante desarrollo, integrada por un equipo multidisciplinar, que cada vez con mayor alcance y especialización, trabaja para identificar estos pacientes y atender sus necesidades. En el año 2010 se atendieron 57 niños y familias y en 2015 la cifra se vio prácticamente duplicada con una suma de 105 pacientes, incluyendo diferentes áreas como consultas externas, hospital de día, urgencias y hospitalización, y también en la comunidad, tanto en el domicilio como en la escuela. Existen diferencias en relación al lugar donde se centran los cui- 
dados y también, respecto al lugar donde fallece el niño. Desde la unidad se intenta fomentar la atención en el domicilio siempre que la situación del paciente, los recursos y la voluntad de la familia sea esa.

Las cifras de mortalidad también se vieron incrementadas en estos 5 años, pasando de 22 pacientes fallecidos en 2010 a 55 en 2015. De los primeros, el $72 \%$ falleció en el hospital y en el año 2015 fue el domicilio el lugar del fallecimiento en el $41 \%$ de los casos.

El lugar de fallecimiento preferido o "ideal" en el caso de los niños ha sido históricamente muy discutido y estudiado sin existir un consenso. Es frecuente extrapolar las preferencias de los adultos a la población pediátrica por lo que la evidencia específica es todavía bastante escasa y proviene mayoritariamente de las impresiones de los padres $(2,7,8,10,11)$. Una revisión sistemática sobre el tema publicada en el año 2013 recoge que ninguno de los dos ámbitos aporta más beneficios en cuanto a la experiencia del fallecimiento, si bien concluye que la oportunidad de planificar el lugar de fallecimiento puede ser mejor indicador de calidad de cuidado al final de la vida, más que el lugar en sí mismo (9). Describen también algunos factores que convierten el domicilio en un lugar más atractivo donde centrar los cuidados y facilitar el fallecimiento, como son: la mayor oportunidad de control del entorno, el estar rodeado de los allegados y familiares, la mayor autonomía y privacidad y el mantenimiento de sentido de normalidad. (13) Es importante puntualizar que el lugar "ideal" es aquel donde el niño y su familia se sientan más cómodos y seguros. En la mayoría de ocasiones, al preguntar a familias y profesionales, el hogar es el lugar elegido (15,16), pero no siempre es el más adecuado. Aspectos como la actuación en momentos de crisis, el no disponer de un soporte de profesional sanitario las 24 horas del día, el manejo de la situación con los hermanos en casa o el miedo al recuerdo del fallecimiento en el hogar, pueden ser elementos que descarten el domicilio como lugar para los últimos días. Si se opta por el hospital para el final de la vida es fundamental proporcionar un ambiente confortable, íntimo y asegurar el acompañamiento durante todo el proceso (7). Algunos autores enfatizan otros factores influyentes como el sexo y la edad $(17,18)$ u otros de la esfera laboral, económica, educacional o social (10). A este nivel, otras investigaciones obtienen resultados muy distintos en poblaciones de características sociales muy similares, concediendo gran importancia a las preferencias culturales y a la disponibilidad de recursos en el territorio $(8,11)$. 


\section{OBJETIVOS}

General:

Conocer qué factores se asocian al fallecimiento en domicilio o en hospital en los pacientes con necesidades paliativas atendidos por la UCPP del hospital Sant Joan de Déu entre 2010 y 2015.

\section{Específicos:}

Analizar la relación entre la cantidad y el ámbito de intervenciones realizadas con el lugar de fallecimiento.

Estudiar si el tiempo de permanencia en la unidad de cuidados paliativos pediátricos se relaciona con el emplazamiento de final de vida (FdV).

Analizar si la especialidad referente del caso se relaciona con el lugar de fallecimiento.

\section{MATERIAL Y MÉTODOS}

Diseño: Estudio descriptivo transversal de tipo retrospectivo

Población: De la muestra inicial de los 205 pacientes que fueron atendidos por la UCPP del Hospital Sant Joan de Déu se seleccionaron aquellos que habían fallecido entre los años 2010 y 2015. Se revisaron las historias clínicas y otros registros de la unidad obteniéndose una muestra final de 198 sujetos.

Criterios de inclusión: niños atendidos por la UCPP en el periodo mencio- nado, independientemente de la edad, sexo, especialidad de procedencia o tiempo y lugar de atención.

Criterios de exclusión: pacientes que no fallecieron en el periodo estudiado.

Las variables estudiadas fueron:

Variables clínicas: Diagnóstico clínico, patología/s y la especialidad referente del caso.

Variables de control: Número de visitas que recibió en domicilio o en hospital, los contactos telemáticos establecidos durante la atención recibida por parte de la UCPP. El tiempo de seguimiento del equipo al niño y su familia.

Variable principal: Lugar de fallecimiento.

Variables sociodemográficas: Edad, sexo.

Se obtuvieron los datos sobre las variables sociodemográficas y relacionadas con la a la atención de los niños y familias de un registro de pacientes creado ad hoc en formato Excel por la unidad de CPP además de historia clínica de cada paciente.

El análisis de datos fue realizado mediante el programa estadístico SPSS Statistics 24.

Las variables cuantitativas como el tiempo de atención por el equipo, la edad, los contactos telemáticos, las visi- 
tas en casa y en el hospital se midieron en medidas de dispersión como las medias y desviación estándar. Las variables cualitativas como el sexo, la especialidad referente del caso, se analizaron con medidas de porcentajes y frecuencias.

Se establecieron relaciones entre diferentes variables para establecer el nivel de dependencia entre ellas y la influencia de unas sobre las otras con tests paramétricos como el test "t"de Student, el coeficiente de correlación de Spearman y Prueba de U Mann-Whitney

Cuestiones éticas: Los autores declaran que no existe ningún conflicto de interés. El estudio ha sido aprobado por parte del Comité de Ética de Investi- gación Clínica (CEIC) del hospital Sant Joan de Déu (Barcelona).

En la realización de este estudio y se han seguido las directrices de confidencialidad y privacidad de la Ley Orgánica de Protección de datos (LOPD). La dirección del centro ha aprobado la realización de este estudio.

\section{REsultados}

De los 198 pacientes incluidos en el estudio, el $51,5 \%$ de los pacientes fueron de sexo masculino y la media de edad al iniciar el seguimiento por la UCPP fue de 7,26 años (rango de 0-272 meses) (ver tabla 1).

\begin{tabular}{|c|c|c|c|c|c|c|c|c|l|}
\hline \multicolumn{2}{|c|}{} & Recuento & Porcentaje (\%) & \multicolumn{2}{|l|}{ Media } & \multicolumn{2}{|l|}{ Frecuencia } & Porcentaje \\
\hline \multirow{2}{*}{ Sexo } & H & 102 & 51,5 & $\begin{array}{c}\text { Tiempo de } \\
\text { seguimiento } \\
\text { (días) }\end{array}$ & 184,71 & $\begin{array}{c}\text { Lugar } \\
\text { exitus }\end{array}$ & Domicilio & 83 & 41,9 \\
\cline { 2 - 10 } & M & 96 & 48,5 & $\begin{array}{c}\text { Edad de } \\
\text { derivación }\end{array}$ & 7,2698 & & Hospital & 115 & 58,1 \\
\hline & Total & 198 & 100,0 & & & Total & 198 & 100,0 \\
\hline
\end{tabular}

Tabla 1. Variables sociodemográficas, tiempo seguimiento y lugar del fallecimiento.

Desde el momento de la derivación a la unidad de cuidados paliativos hasta la muerte la media de tiempo fue de 184 días (intervalo de confianza de $95 \%)$ siendo el rango de tiempo de seguimiento de 0 a 1204 días (ver tabla 1). El $50 \%$ de los casos fueron derivados desde oncología y las especialidades nooncológicas mayoritarias de referencia fueron neurología $(28,3 \%)$ seguida por pediatría $(7,6 \%)$ y neonatología $(4,5 \%)$. El $41,9 \%$ de los pacientes murieron en su domicilio (ver tabla 2). 


\begin{tabular}{|c|c|c|}
\hline Especialidad & Frecuencia & Porcentaje \\
\hline Cardiología & 3 & 1,5 \\
\hline Crónicos & 2 & 1,0 \\
\hline Endocrino & 1 & 0,5 \\
\hline Gastroenterología & 3 & 1,5 \\
\hline Hematología & 8 & 4,0 \\
\hline Neonatología & 9 & 4,5 \\
\hline Neurocirugía & 2 & 1,0 \\
\hline Neurología & 56 & 28,3 \\
\hline Oncología & 99 & 50,0 \\
\hline Pediatría & 15 & 7,6 \\
\hline Total & 198 & 100,0 \\
\hline & & \\
\hline
\end{tabular}

Tabla 2. Especialidad médica que deriva al paciente a la UCPP.

Se estudió la distribución del lugar de fallecimiento del niño según la especialidad médica referente: los pacientes afectos de patología oncológica fallecen mayoritariamente en el hospital $(62,6 \%)$ representando el $54 \%$ del total de las muertes que suceden en este contexto. Lo mismo ocurrió con aquellos con alguna enfermedad neurológica grave, aunque se reparte de forma más simétrica en hospital y domicilio (Ver figura 1).

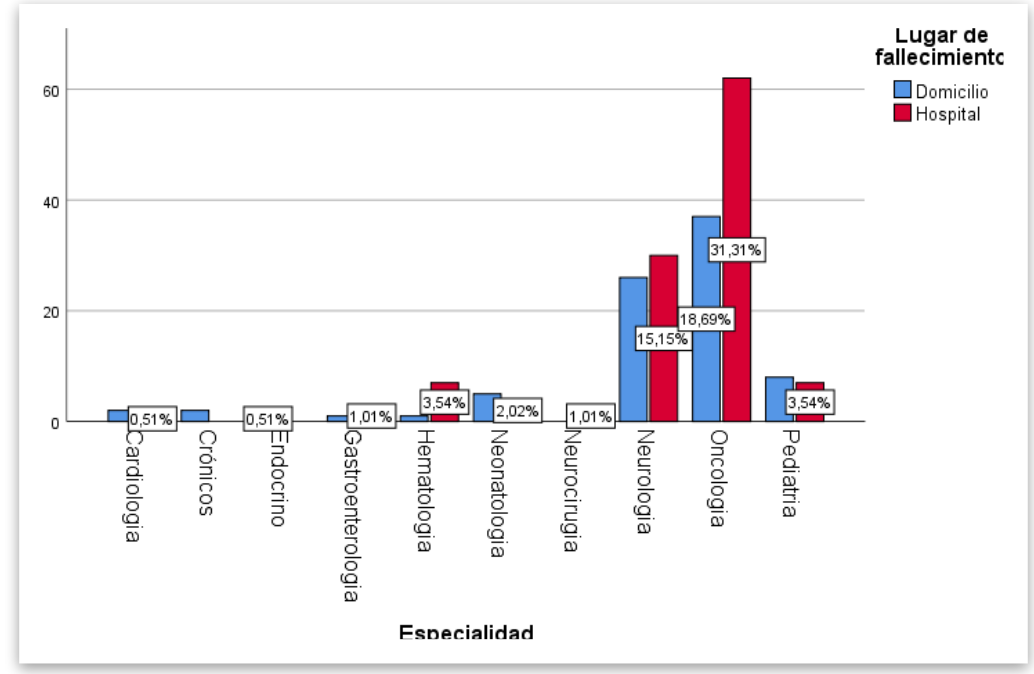

Figura 1. Proporción de pacientes por lugar de fallecimiento relacionado con especialidad.

El coeficiente de correlación entre el número de consultas domiciliarias y telefónicas fue de 0,812; también existe correlación positiva entre el tiempo de permanencia en la unidad y las consultas domiciliarias $(p=0,564)$ y telefónicas $(p=0,568)$ (ver tabla 3). La correlación entre las visitas en consulta externa y las 


\section{llamadas de seguimiento presenta una vada significación estadística.}

significación bilateral de 0,009, con ele-

\begin{tabular}{|c|c|c|c|c|c|c|}
\hline \multicolumn{7}{|c|}{ Correlaciones } \\
\hline & & & $\begin{array}{c}\text { Tiempo } \\
\text { seguimiento }\end{array}$ & $\begin{array}{c}\text { Consultas } \\
\text { hospital }\end{array}$ & $\begin{array}{c}\text { Consultas } \\
\text { domiciliarias }\end{array}$ & $\begin{array}{l}\text { Consultas } \\
\text { telefónicas }\end{array}$ \\
\hline \multirow[t]{12}{*}{$\begin{array}{l}\text { Rho de } \\
\text { Spearman }\end{array}$} & \multirow[t]{3}{*}{$\begin{array}{c}\text { Tiempo } \\
\text { seguimiento }\end{array}$} & $\begin{array}{c}\text { Coeficiente de } \\
\text { correlación }\end{array}$ & 1,000 & ,076 &, $564^{* *}$ &, $568^{* *}$ \\
\hline & & Sig. (bilateral) & . & 291 & , 000 & ,000 \\
\hline & & $\mathrm{N}$ & 198 & 197 & 186 & 194 \\
\hline & \multirow[t]{3}{*}{$\begin{array}{c}\text { Consultas } \\
\text { hospital }\end{array}$} & $\begin{array}{c}\text { Coeficiente de } \\
\text { correlación }\end{array}$ & , 076 & 1,000 & , 020 & , $184^{* *}$ \\
\hline & & Sig. (bilateral) & 291 & . & 784 & ,009 \\
\hline & & $\mathrm{N}$ & 197 & 204 & 192 & 200 \\
\hline & \multirow[t]{3}{*}{$\begin{array}{c}\text { Consultas } \\
\text { domiciliarias }\end{array}$} & $\begin{array}{c}\text { Coeficiente de } \\
\text { correlación }\end{array}$ &, $564^{* *}$ & , 020 & 1,000 & ,812* \\
\hline & & Sig. (bilateral) & ,000 & 784 & & ,000 \\
\hline & & $\mathrm{N}$ & 186 & 192 & 193 & 193 \\
\hline & \multirow[t]{3}{*}{$\begin{array}{l}\text { Consultas } \\
\text { telefónicas }\end{array}$} & $\begin{array}{c}\text { Coeficiente de } \\
\text { correlación }\end{array}$ & ,568* & , 184* & ,812** & 1,000 \\
\hline & & Sig. (bilateral) & ,000 & ,009 & ,000 & . \\
\hline & & $\mathrm{N}$ & 194 & 200 & 193 & 201 \\
\hline
\end{tabular}

Tabla 3. Coeficiente de correlación de Spearman entre variables cuantitativas

\begin{tabular}{|c|c|c|c|c|}
\hline \multicolumn{5}{|c|}{ Prueba de Mann-Whitney } \\
\hline & Especialidades agrupadas & $\mathrm{N}$ & $\begin{array}{c}\text { Rango } \\
\text { promedio }\end{array}$ & $\begin{array}{c}\text { Suma de } \\
\text { rangos }\end{array}$ \\
\hline \multirow[t]{3}{*}{ Consultas hospitalarias } & Hemato-Onco & 110 & 121,94 & 13413,50 \\
\hline & Otros & 94 & 79,75 & 7496,50 \\
\hline & Total & 204 & & \\
\hline \multirow[t]{3}{*}{ Consultas domiciliarias } & Hemato-Onco & 106 & 101,04 & 10710,50 \\
\hline & Otros & 87 & 92,07 & 8010,50 \\
\hline & & 193 & & \\
\hline \multirow[t]{3}{*}{ Consultas telefónicas } & Hemato-Onco & 110 & 103,37 & 11371,00 \\
\hline & Otros & 91 & 98,13 & 8930,00 \\
\hline & Total & 201 & & \\
\hline \multirow[t]{3}{*}{ Tiempo seguimiento } & Hemato-Onco & 107 & 114,35 & 12235,00 \\
\hline & Otros & 91 & 82,04 & 7466,00 \\
\hline & Total & 198 & & \\
\hline
\end{tabular}

Tabla 4. Prueba de U Mann-Whitney 
Se dividieron las especialidades en dos grupos de onco-hematología $u$ otra especialidad obteniéndose diferencias estadísticamente significativas $(p<0,001)$ entre ambos a nivel del número de consultas hospitalarias, domiciliarias, telefónicas y el tiempo de seguimiento de CPP (ver tabla 4).

La media de consultas hospitalarias fue de 20,94 (desviación típica 19,03) y 8,22 (desviación típica 11,28) en los niños que fallecieron en el hospital y domicilio respectivamente (Figura 2).

La media del número de visitas en domicilio fue de 8,11 y telefónicas 31,17 en los niños fallecidos en domicilio. Sin embargo, los niños que fallecieron en ámbito hospitalario recibieron de media 1,57 visitas domiciliarias y 14,66 telemáticas (ver figuras 3 y 4 ).

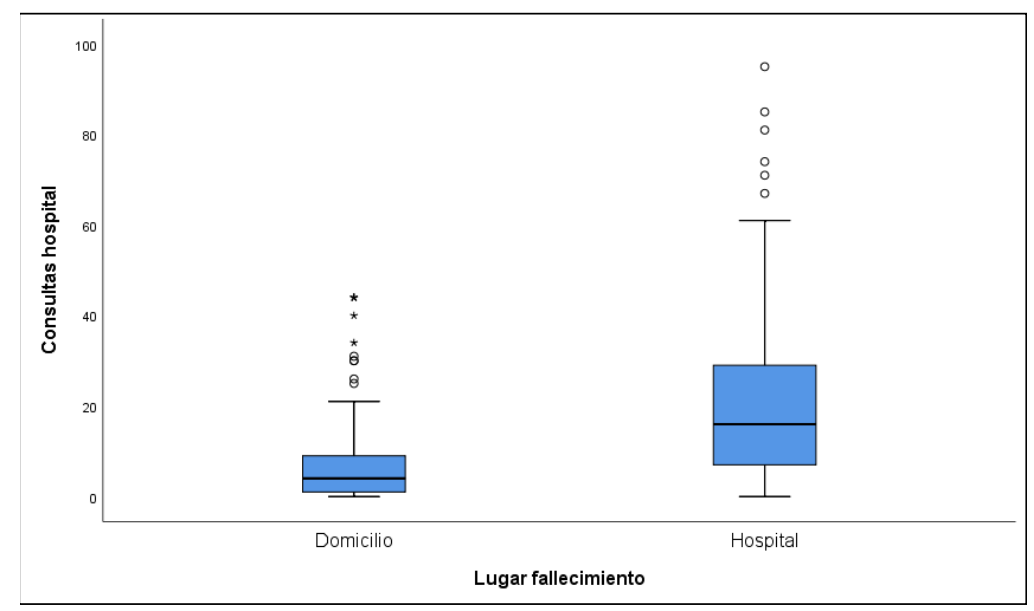

Figura 2. Número de consultas hospitalarias según lugar de fallecimiento

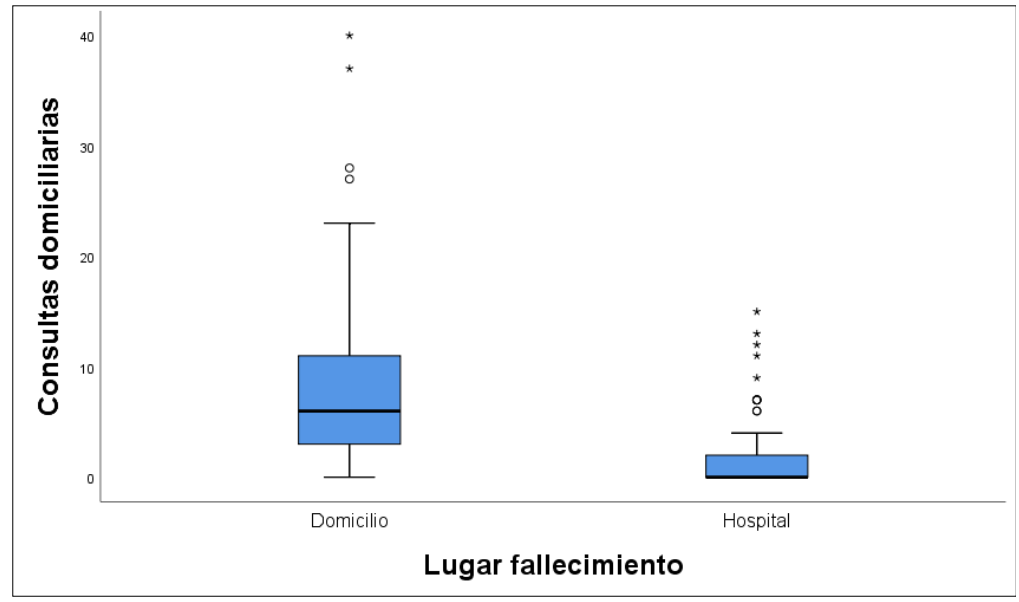

Figura 3. Número de consultas domiciliarias según lugar de fallecimiento 


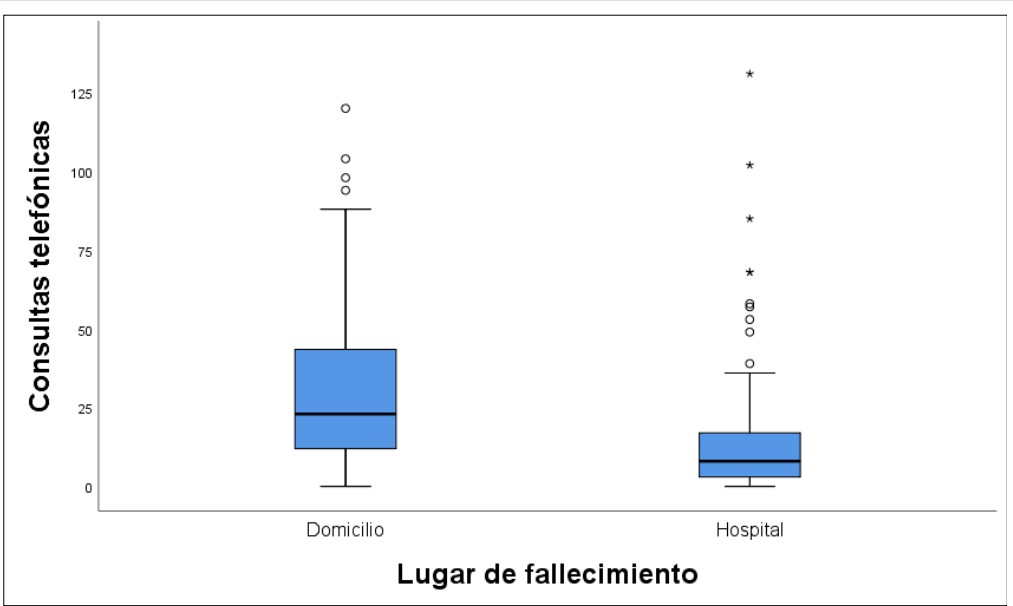

Figura 4. Número de consultas telefónicas según lugar de fallecimiento.

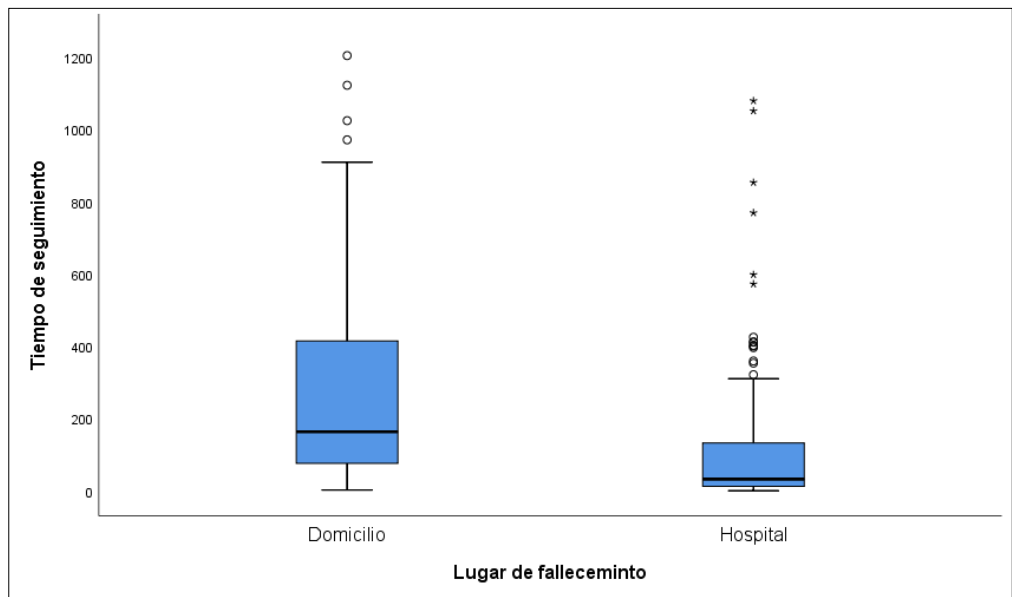

Figura 5. Tiempo de seguimiento (días) en pacientes que fallecieron en domicilio u hospital.

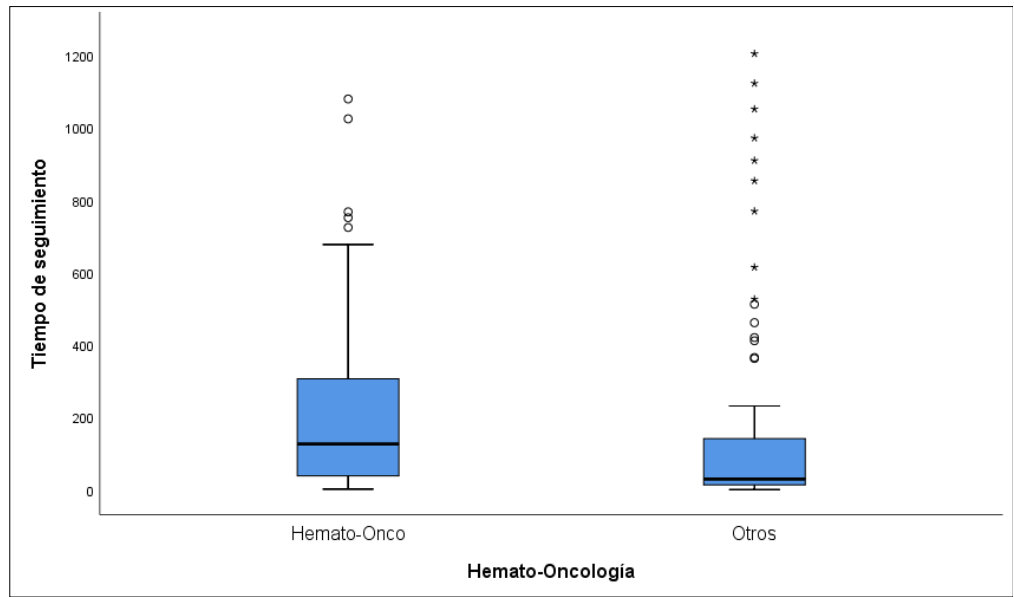

Figura 6. Tiempo (días) de permanencia en UCPP según especialidad de derivación agrupada 


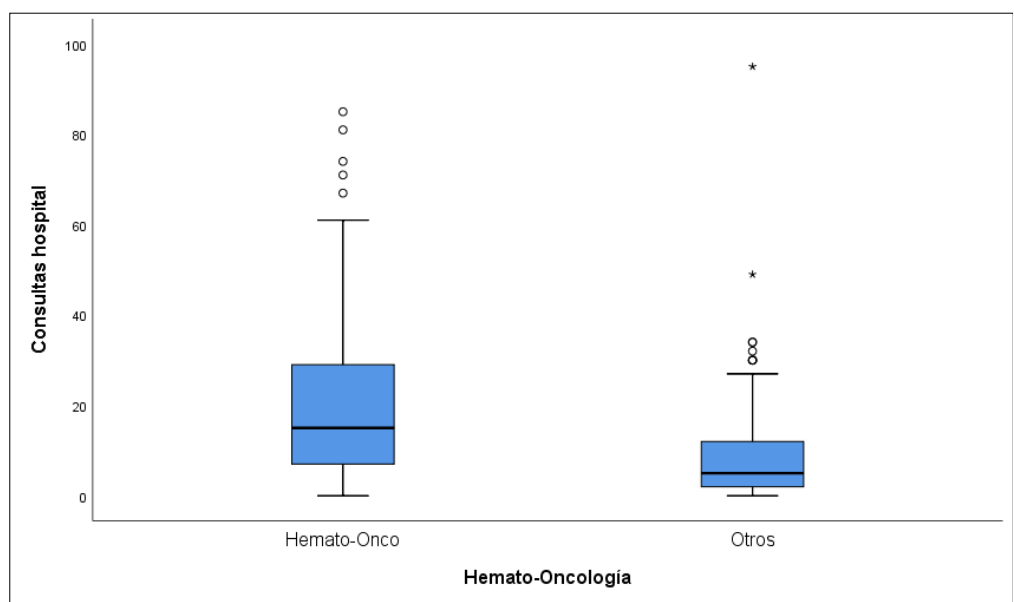

Figura 7. Media de consultas hospitalarias por especialidad originaria

El tiempo medio de seguimiento en la unidad fue de 264 días en los niños que fallecieron en su domicilio frente a los 201 de aquellos que lo hicieron en el hospital (ver gráfico 5).

Este periodo de seguimiento en el grupo de pacientes con patología onco -

\section{Discusión}

En el presente trabajo se proporciona una muestra notablemente representativa de la población de niños de toda la comunidad autónoma (Cataluña) con necesidades paliativas, ya que se trata de un centro de referencia y el único que en el momento del estudio, contaba en su cartera de servicios con atención domiciliaria y específica en el final de vida de los niños que fallecen por causa previsible.

En la experiencia de la muestra estudiada dos tercios de los pacientes con patología onco-hematológica falle- hematológica es de 209 días de media con respecto a los 155 días en el grupo de especialidades no onco-hematológicas y la media de consultas hospitalarias es de 20,15 y de 9,72 respectivamente (ver figuras 6 y 7 ).

cen en el hospital, si bien no se han encontrado diferencias estadísticamente significativas en la distribución del lugar de fallecimiento comparándolos con el resto de especialidades. Este estudio difiere de gran parte de la bibliografía, que reporta que los pacientes afectos de algún tipo de cáncer en cuidados paliativos son más susceptibles de morir en casa. En la revisión bibliográfica del 2013, cuya muestra final incluye 9 artículos de los cuales 6 se centraban en pacientes con cáncer y destacaban la preferencia mayoritaria de los padres del domicilio para el final de la vida y el fallecimiento. Evalúan también cómo se produce un cambio de preferencia hacia el 
fallecimiento en casa en lugar del hospital a medida que pasa el tiempo durante las últimas semanas de vida.

En la misma línea, al estudiar otras variables como el tiempo de seguimiento en uno u otro grupo de especialidad, se detecta un mayor tiempo de seguimiento en las enfermedades oncohematológicas. Se obtuvieron diferencias en la distribución del número de consultas hospitalarias, domiciliarias, telefónicas y el tiempo de permanencia en el programa de CPP entre los grupos de enfermedad onco-hematológica y no onco-hematológica, con alta significación estadística.

En gran parte de los estudios revisados, no se incluían niños en el período perinatal ni neonatal $(9,12)$ lo cual ha sido ampliado en este estudio, incluyendo los pacientes desde los 0 días de vida y obteniendo que los pacientes derivados desde la unidad de neonatología y desde la unidad de pediatría general mueren en mayor proporción en su domicilio.

En cuanto a la atención provista por parte de la UCPP, las consultas hospitalarias fueron más numerosas en los niños que fallecieron en el hospital mientras que el número de visitas comunitarias y telemáticas fue mayor en aquellos que murieron en casa, de la misma forma que el tiempo de seguimiento en la unidad. Esto podría justificarse por la oportunidad que proporciona el tiempo para establecer un vínculo de confianza y una adecuada relación terapéutica que permita conocer a al paciente y su familia, explorar sus deseos y empoderarlos para vivir el fallecimiento del niño en el hogar, si es el lugar que ellos prefieren (9).

A nivel de la trayectoria de enfermedad, sería incorrecto inferir que los resultados de estudios sobre preferencia del lugar de fallecimiento basados en población con una única enfermedad o una enfermedad como el cáncer, que tienen mayor representación en la literatura, puedan generalizarse a todas las enfermedades amenazantes o limitantes para la vida ${ }^{(9)}$.

En un trabajo reciente sobre las características del paciente y lugar de fallecimiento se analizó el código diagnóstico (CIE-10) y el grupo de enfermedad clasificada por la Association for Children with Terminal conditions (ACT), concluyendo que las necesidades de cuidado y la supervivencia son significativamente diferentes entre los cuatro grupos ACT (8). Es lógico pensar que, al tratarse de una unidad de atención domiciliaria específica en este caso, la mayor parte de defunciones ocurrieran en el hogar; vale la pena destacar que el $96 \%$ de los pacientes incluidos en este artícu- 
lo, fallecieron allí donde lo deseaban. Resultaría interesante, entonces, relacionar los factores analizados en este estudio con otras características como pueden ser los grupos de trayectorias de enfermedades de la ACT (1), los días de ingreso de los pacientes que fallecen en el hospital y la calidad de vida relacionada con la salud percibida por ellos mismos y/o sus familias.

En la gran mayoría de la bibliografía revisada, concluyen que el lugar de fallecimiento no es uno de los ítems más relevantes en la experiencia ni más relacionados con la calidad del final de la vida per se, sino que enfatizan la importancia de fallecer donde se deseaba y la oportunidad de anticipar y planificar adecuadamente el final de la vida (21).

Los resultados alcanzados pueden no ser extrapolables a todos los niños con necesidades paliativas y/o en el final de sus vidas, ya que existen numerosos otros factores influyentes como pueden ser los recursos de atención comunitaria disponibles, la sensibilización de otros profesionales para la identificación y derivación precoz de los pacientes o la tipología de los mismos, que ocasionarían probablemente, cambios significativos en los resultados.

Dado el aumento de población pediátrica identificada como subsidiaria de recibir cuidados paliativos, el incipien- te desarrollo de la unidad en recursos y población diana y la sensibilización de los profesionales que motiva una derivación precoz y específica a CPP, sería interesante poder replicar este estudio y compararlo con las características y datos recogidos en la actualidad.

Sería interesante para futuros estudios incluir si el niño y la familia lograron vivir los últimos días allí donde ellos deseaban.

\section{CONCLUSIONES}

Los resultados encontrados indican que un mayor tiempo de atención por el equipo de CPP se asocia con el fallecimiento en domicilio, al igual que el número mayor de consultas domiciliarias y telefónicas. Esto permite establecer que a mayor tiempo recibiendo atención paliativa por parte de la unidad, las familias de un niño en proceso de enfermedad avanzada, aumentan su confianza en el equipo y su nivel de autonomía y competencia en los cuidados, favoreciendo que asuman el fallecimiento del niño en el hogar. Todo esto disminuye el número de visitas al hospital al mismo tiempo que aumenta el número de visitas en la comunidad y/ o consultas telemáticas.

En cuanto a la relación entre el número de visitas en hospital y la especialidad referente se confirma la 
hipótesis planteada al inicio del estudio de que la especialidad médica que deriva al niño a cuidados paliativos condiciona un determinado estilo de cuidados y afrontamiento más o menos dependiente del entorno hospitalario como zona de seguridad, lo cual implica mayor presencia (mayor número de consultas) de estos especialistas de referencia durante el proceso de enfermedad avanzada.

\section{AGRADECIMIENTOS}

Al equipo de profesionales de cuidados paliativos pediátricos del HSJD por permitir el acceso al registro elaborado durante su desarrollo a lo largo de los últimos años, que ha permitido la realización de este trabajo y por su apoyo incondicional al proyecto.

Transmitir el agradecimiento también al equipo de soporte a la investigación enfermera del Hospital Sant Joan de Déu por el asesoramiento continuado y al soporte estadístico del hospital por su ayuda en la fase analítica de los datos. 


\section{BIBLIOGRAFÍA}

1. Chambers L, ACT-Association for Children's Palliative Care. A guide to the development of children's palliative care services. Bristol: ACT; 2009.

2. Grupo de trabajo de Cuidados Paliativos para niños de la EAPC. Cuidados paliativos para lactantes, niños, y jóvenes: Los Hechos. [Internet]. Fondazione Maruzza Lefevbre D’Ovidio Onlus.Roma. 2009

3. Craig, F., Huijer, A. S., Benini, F., Kuttner, L., Wood, C., Ferraris, P. C., \& Al., E. (2007). IMPaCCT: standards for paediatric palliative care in Europe. European Association for Palliative Care. Eur J Palliat Care, 14(3), 109-16.

4. World Health Organization. Cancer pain relief and palliative care in children. Geneva: WHO, 1998

5. American Academy of Pediatrics. Committee on Bioethics and Committee on Hospital Care. Palliative Care for Children. Pediatrics.2000; Vol.106 (2).

6. Ministerio de Sanidad, Servicios Sociales e Igualdad. Sanidad 2014. Cuidados paliativos pediátricos en el Sistema Nacional de Salud: Criterios de Atención.

7. Navarro S, Martino R. Cuidados Paliativos Pediátricos: Epidemiología, organización. Fallecimiento en el hospital. Sedación. Actual Sephoes. 2013;25

8. Bender, H. U., Riester, M. B., Borasio, G. D., \& Fuhrer, M. (2017). "Let's Bring Her Home First." Patient Characteristics and Place of Death in Specialized Pediatric Palliative Home Care. Journal of Pain and Symptom Management, 54(2), 159-166.

9. Bluebond-Langner, M., Beecham, E., Candy, B., Langner, R., \& Jones, L. (2013). Preferred place of death for children and young people with life-limiting and life-threatening conditions: A systematic review of the literature and recommendations for future inquiry and policy. Palliative Medicine, 27(8), 705-713.

10. Kurashima, A., Latorre, M., Teixeira, S., \& de Camargo, B. (2005). Factors associated with location of death of children with cancer in palliative care. Palliative and Supportive Care, 3(2), 115-119.

11. Håkanson, C., Öhlén, J., Kreicbergs, U., Cardenas-turanzas, M., Wilson, D. M., Loucka, M.,Naylor, W. (2017). Place of death of children with complex chronic conditions: cross-national study of 11 countries, 327-335. Eur J Pediatr. 2017 Mar;176(3):327-335.

12. Chang E, MacLeod R, Drake R Characteristics influencing location of death for children with lifelimiting illness Archives of Disease in Childhood 2013;98:419-424

13. Kassam, A., Skiadaresis, J., Alexander, S. and Wolfe, J. (2014), Parent and clinician preferences for location of end-of-life care: Home, hospital or freestanding hospice?. Pediatr Blood Cancer, 61: 859-864.

14. Feudtner C, Feinstein JA, Satchell M, Zhao H, Kang TI. Shifting Place of Death Among Children With Complex Chronic Conditions in the United States, 1989-2003. JAMA. 2007;297(24):27252732.

15. Townsend, J., Frank, A., Fermont, D., Dyer, S., Karran, O., \& Walgrove, A. (1990). Terminal cancer care and patients ' preference for place of death : a prospective study, 301(September), 415-417.

16. Karlsen, S., \& Lecturer, J. A. S. (1998). How do cancer patients who die at home differ from those who die elsewhere? 6591(98), 279-286.

17. Bruera, E., Neumann, C.M., Gagnon, B., et al. 1999. Edmonton Regional Palliative Care Program: Impact on patterns of terminal cancer care. CMAJ, 10, 161 3 290-293.

18. Grande, G.E., Addington-Hall, J.M., \& Todd, C.J. 1998. Place of death and access to home care services: are certain patient groups at a disadvantage? Soc Sci Med., 47 5 565-579. Review.

19. Mack, J. W., Hilden, J. M., Watterson, J., Moore, C., Turner, B., Grier, H. E. Wolfe, J. (2005). Parent and physician perspectives on quality of care at the end of life in children with cancer. Journal of Clinical Oncology, 23(36), 9155-9161.

20. Gethins, M. (2012). Pediatric palliative care in Europe expands. Journal of the National Cancer Institute, 104(1), 10-11.

21. Dussel, V., Kreicbergs, U., Hilden, J. M., Watterson, J., Moore, C., Turner, B. G. Wolfe, J. (2009). Looking Beyond Where Children Die: Determinants and Effects of Planning a Child's Location of Death. Journal of Pain and Symptom Management, 37(1), 33-43. 